\title{
O089: Recurrent transmission of group a streptococcus pyogenes (GAS) during surgery by a health care worker $(\mathrm{HCW})$
}

\author{
C Landelle ${ }^{1 *}$, P Lesprit ${ }^{1}$, P Legrand ${ }^{2}$, P Brehaut ${ }^{1}$, D Ducellier ${ }^{1}$, J-F Papon ${ }^{3}$, E Allaire ${ }^{4}$, E Girou ${ }^{1}$, J-P Becquemin ${ }^{4}$, \\ C Brun-Buisson ${ }^{5}$
}

From 2nd International Conference on Prevention and Infection Control (ICPIC 2013)

Geneva, Switzerland. 25-28 June 2013

\section{Introduction}

Surgical site infections (SSI) due to GAS are rare but potentially life-threatening.

\section{Objectives}

We describe 2 cases occurring after thyroidectomy in two female patients (aged 36 and 52 years respectively), who developed septic shock with multi-organ failure, mediastinitis and empyema, respectively 2 and 4 days after surgery performed 4 months apart (Nov. 2009; Feb. 2010).

\section{Methods}

We interviewed patients or relatives and operating room (OR) personnel with regard to recent throat infection, and investigated SSI prevention measures, surgical masks wearing in the OR, and GAS carriage by HCWs; GAS isolates were compared by molecular typing.

\section{Results}

There was no recent history of throat infection in patients or their relatives. Compliance to SSI preventive measures (pre operative showers, skin antisepsis, and laminar air flow) was adequate, and wearing of mask in the OR was adequate for $88 \%$ of $332 \mathrm{HCWs}$. A GAS isolate was recovered from throat swabs of 2 of the $6 \mathrm{HCWs}$ caring for the $1^{\text {st }}$ case, one of which was identical to the patient's isolate. Auditing this HCW revealed a lack of adequate fitting of the mask during preparation of the OR. Educational sessions were implemented. After the $2^{\text {nd }}$ case occurred, the same HCW was again found colonized with a GAS isolate

\footnotetext{
${ }^{1}$ Unité de Contrôle, Epidémiologie et Prévention de I'Infection, CHU Albert Chenevier-Henri Mondor, Assistance Publique-Hôpitaux de Paris; Université Paris 12, Créteil, France

Full list of author information is available at the end of the article
}

identical to the patient's isolate, but different from the $1^{\text {st }}$ one. A more in-depth investigation revealed that one of his children had recurrent tonsillitis. Decolonization of the $\mathrm{HCW}$ was attempted, but GAS carriage recurred until tonsillectomy was performed on his child.

\section{Conclusion}

The same staff carrier was involved in the transmission of 2 different GAS strains, likely resulting from household transmission. Throat carriage of the personnel stopped only after tonsillectomy of his child. Reinforcing adequate surgical mask wearing in the OR is important, but $100 \%$ compliance appears difficult to maintain.

\section{Disclosure of interest}

None declared.

\begin{abstract}
Author details
${ }^{1}$ Unité de Contrôle, Epidémiologie et Prévention de I'Infection, CHU Albert Chenevier-Henri Mondor, Assistance Publique-Hôpitaux de Paris; Université Paris 12, Créteil, France. ${ }^{2}$ Service de Bactériologie-Virologie-Hygiène, CHU Albert Chenevier-Henri Mondor, Assistance Publique-Hôpitaux de Paris; Université Paris 12, Créteil, France. ${ }^{3}$ Service d'Oto-Rhino-Laryngologie, CHU Albert Chenevier-Henri Mondor, Assistance Publique-Hôpitaux de Paris; Université Paris 12, Créteil, France. ${ }^{4}$ Service de Chirurgie Vasculaire et Endocrinienne, CHU Albert Chenevier-Henri Mondor, Assistance PubliqueHôpitaux de Paris; Université Paris 12, Créteil, France. ${ }^{5}$ Service de Réanimation Médicale, CHU Albert Chenevier-Henri Mondor, Assistance Publique-Hôpitaux de Paris; Université Paris 12, Créteil, France.
\end{abstract}

Published: 20 June 2013

doi:10.1186/2047-2994-2-S1-089

Cite this article as: Landelle et al: O089: Recurrent transmission of group a streptococcus pyogenes (GAS) during surgery by a health care worker (HCW). Antimicrobial Resistance and Infection Control 2013 2(Suppl 1):089.

\section{Ciomed Central}

(c) 2013 Landelle et al; licensee BioMed Central Ltd. This is an Open Access article distributed under the terms of the Creative Commons Attribution License (http://creativecommons.org/licenses/by/2.0), which permits unrestricted use, distribution, and reproduction in any medium, provided the original work is properly cited. 